\title{
Reproductive Endocrinology
}

\author{
Bruno Ferraz-de-Souzaa, Lin Lina, Teresa K. Woodruff ${ }^{b}$ and John C. Achermann ${ }^{a}$ \\ a UCL Institute of Child Health, University College London, London, UK \\ bNorthwestern University, Chicago, III., USA
}

\begin{abstract}
This past year has seen ongoing intense debate about regeneration of ovarian germ cells, potential advances in fertility preservation in males and females, and important cross-sectional studies - all from the University of Copenhagen - on precocious puberty, hypospadias incidence and anatomical correlates of the postnatal testosterone surge. Whilst many of the new technologies might seem one step removed from daily practice, it is becoming essential that patients and their families receive appropriate and insightful disclosure about their conditions and potential therapeutic options in the future. The papers of Sutton et al. and Minto et al. from the adult clinics demonstrate this well, and highlight the importance of pediatricians getting this right in individuals with Turner syndrome or disorders of sex development.
\end{abstract}

\section{Mechanism of the year: keeping abreast of stem cell research}

\section{Generation of a functional mammary gland from a single stem cell}

Shackleton M, Vaillant F, Simpson KJ, Stingl J, Smyth GK, Asselin-Labat ML, Wu L, Lindeman GJ, Visvader JE The Walter and Eliza Hall Institute of Medical Research, Parkville, Vic., Australia

visvader@wehi.edu.au

Nature 2006;439:84-88

Background: It has long been postulated that mammary stem cells (MaSCs) exist, given the regeneration of mammary tissue seen following transplantation of epithelial fragments in mice. However, the identity and purification of MaSCs has proved elusive owing to the lack of defined markers.

Methods: Discrete populations of mouse mammary cells were isolated on the basis of cell-surface markers. A subpopulation (Lin-CD29hiCD24+) highly enriched for MaSCs by transplantation was studied further.

Results: Using cells from a LacZ transgene expressing mouse, it was shown that a single putative MaSC from this population was able to reconstitute a complete mammary gland in vivo. This gland contained both luminal and myoepithelial lineages and generated functional lobuloalveolar units during pregnancy.

Conclusions: Single cells within the Lin-CD29hiCD24+ population are likely to represent MaSCs and can generate functional breast tissue.

The potential benefits of stem cell research are being gradually realized, but the application of these new technologies to human disease treatment poses many challenges. It was reassuring, therefore, to see this report of the identification of putative mammary stem cells (MaSCs) and, more importantly, the generation of entirely functional multi-lineage breast tissue that contains both ductal and lobular structures from a single precursor stem cell. The hierarchical process of self-renewal and differentiation were explored further in a second Nature paper by this research team shortly after [1]. Does this have relevance to humans? Certainly these are potentially important findings for the study of tumorigenesis, as breast cancers contain a high proportion of these putative MaSCs and the origin of the cancer stem cell remains elusive. Furthermore, there may be benefits for breast augmentation in girls where estrogenic treatment has been delayed or resulted in poor cosmesis. Most importantly, however, it shows that - in the mouse at least - functional whole tissue regeneration of an 'endocrine' organ can be done! 
The following series of original papers, commentaries and reviews summarizes one of the most contentious debates in reproductive endocrinology.

\title{
Oocyte generation in adult mammalian ovaries by putative germ cells in bone marrow and peripheral blood
}

\author{
Johnson J, Bagley J, Skaznik-Wikiel M, Lee HJ, Adams GB, Niikura Y, Tschudy KS, Tilly JC, Cortes ML, Forkert R, \\ Spitzer T, lacomini J, Scadden DT, Tilly JL \\ Vincent Center for Reproductive Biology, Vincent Obstetrics and Gynecology Service, Harvard Medical School, \\ Boston, Mass., USA \\ jtilly@partners.org \\ Cell 2005;122:303-315
}

Background: The prevailing dogma of mammalian reproduction states that females lose the capacity to produce oocytes during fetal development and are born with a finite number of oocytes, of which only a small number will be ovulated after puberty. A recent study suggests that juvenile and adult mice are capable of regenerating oocytes after treatment with sterilizing chemotherapeutic agents, though the existence of germline stem cells, but their origin had not been proven.

Methods: The expression of multiple germline markers was detected in the bone marrow and peripheral blood of adult female mice using Northern blot analysis, immunohistochemistry, and PCR. Bone marrow and peripheral blood collected from adult female mice was transplanted, via injection into the tail vein, into recipient adult female mice that had been sterilized by treatment with the chemotherapeutic agents cyclophosphamide and busulfan.

Results: Two months after chemotherapy, the ovaries of sterilized mice that received bone marrow transplantation had several hundred oocyte-containing follicles at all stages of development that persisted for more than 11 months after transplantation. Similar results were reported for ataxia telangienctasia-mutated (Atm) null mice that underwent chemotherapy; these mice do not produce mature germ cells and are born with no oocytes. In a second set of experiments, peripheral blood was collected from transgenic female mice containing a GFP reporter driven by the germ cell-restricted Oct4 promoter and injected into chemotherapy-sterilized wild-type or Atm null female mice. GFP-positive oocytes were detected in the recipient mice within $28-30 \mathrm{~h}$ of peripheral blood transplantation.

Conclusion: Germline stem cells originating in the bone marrow are capable of regenerating oocytes in the ovaries of mice sterilized by chemotherapy, although the competency of these oocytes to be fertilized and produce viable offspring is unknown.

This paper showing putative oocyte regeneration from germ cells in the bone marrow, along with its predecessor published in Nature 2004 showing potential follicular renewal in the postnatal ovary [2], presented one of the most controversial challenges to prevailing dogma in female reproductive biology. Until recently it has been believed that the number of oocytes is fixed in early development. In humans, less than $3 \times 10^{5}$ of the original pool of about $10^{6}$ non-degenerative oocytes present at birth survive at puberty. This number continues to decline throughout adulthood to the point of exhaustion around age 50, driving menopause. These papers have challenged this view by suggesting that the oocyte population is renewable. Their publication sparked furious debate that continues today (see below). If the findings of Johnson et al. that putative BM-derived germ cells are capable of sustaining long-term oocyte production are proven correct, these data have profound implications in not only the possibility for recovery of fertility after sterilizing chemotherapy, but also with regard to the mechanism by which loss of fertility occurs at menopause and the link between reproductive aging and the loss of stem cell viability over the lifespan. It is important to emphasize that this paper does not prove that oocytes produced as a result of BMT are competent for fertilization, embryonic development, and the generation of viable offspring. Nevertheless, these findings would represent a paradigm shift in our view of oocyte biology, and may also pave the way for potential germ cell recovery in males with spermatogenic failure. 


\title{
Editorial: on regenerating the ovary and generating controversy
}

Telfer EE, Gosden RG, Byskov AG, Spears N, Albertini D, Andersen CY, Anderson R, Braw-Tal R, Clarke H, Gougeon A, McLaughlin E, McLaren A, McNatty K, Schatten G, Silber S, Tsafriri A Institute of Cell Biology, School of Biological Sciences, University of Edinburgh, UK evelyn.telfer@ed.ac.uk

cell 2005;122:821-822

Johnson et al.'s challenge to the time-honored concept that oogenesis is restricted to fetal development understandably sparked controversy and led to a number of editorials. Telfer et al.'s editorial, written by 16 leading reproductive biologists, questioned the validity of data reported in Nature in 2004 and in Cell in 2005. With regard to the 2004 paper [2], Telfer et al. contended that the reported results were open to alternate explanations and that Johnson et al. had since reversed their initial hypothesis that germline stem cells may reside in the ovarian surface epithelium. With regard to the 2005 paper, the main arguments made were that: (1) the results have not been replicated by others; (2) the authors had not sufficiently verified the identity or origin of germline stem cells or their competency to undergo fertilization and subsequent embryonic development; (3) oogenesis cannot be completed within $24 \mathrm{~h}$ as reported and it is unclear whether this accelerated oogenesis would impact fertilization; (4) the germline markers identified in the bone marrow are also expressed in other cells and organs; (5) detection of GFP-positive oocytes in recipient ovaries following peripheral blood transplantation may not necessarily support the existence of germline stem cells in the bone marrow, and (6) the authors have not described the mechanism by which bone marrow stem cells are able to develop into oocytes in the ovary. Telfer et al. suggested several additional experiments to prove the lineage of 'new' oocytes, and concluded by expressing concern that extrapolation of the results of the Johnson et al. studies to humans was premature.

\section{Setting the record straight on data supporting postnatal oogenesis in female mammals}

\author{
Johnson J, Skaznik-Wikiel M, Lee HJ, Niikura Y, Tilly JC, Tilly JL \\ Vincent Center for Reproductive Biology, Vincent Obstetrics and Gynecology Service, Massachusetts General \\ Hospital/Harvard Medical School, Boston, Mass., USA \\ jtilly@partners.org \\ Cell Cycle 2005;4:1471-1477
}

Background: In this article, Johnson et al. responded to several criticisms that appeared in four separate commentaries regarding their findings on postnatal oogenesis reported in Nature in 2004 and Cell in 2005, specifically focusing on the criticisms raised by Telfer et al. in their commentary in Cell (see above).

Johnson et al. methodically responded to each criticism raised by Vogel in Science [3], Ainsworth in Nature [4], Powell in Nature Medicine [5], and Telfer et al. in Cell. First, the authors contended that postnatal regeneration of oocytes had been described as early as the $1920 \mathrm{~s}$ and continued until the publication of Zuckerman et al.'s paper in 1951. Since then, the concept of a fixed number of oocytes that exists at birth has been accepted without challenge, primarily because the ovary was thought to be the only source of germline stem cells. Johnson et al. went on to agree that there have been no studies that have confirmed their results, but also pointed out that there have been no studies that refute them. They argued that their follow-up Cell paper confirmed and strengthened many of the original hypotheses reported in the Nature paper but do not directly address the difference in origins (ovarian cortical cells vs. bone marrow-derived). The authors also defended their mouse models, stating that germline stem cells within the recipient ovary could not have produced the new oocytes that developed after chemotherapy in wild-type or Atm null mice, as these models simply do not contain any oocytes or developing follicles. The authors cited specific references in the literature to defend their use of Atm null mice as a recipient model that always lacks oocytes and developing follicles, as well as their use of selected markers to identify germline stem cells in the bone marrow. They also defended their use of known oocyte markers and histology to confirm the existence of new oocytes within the recipient ovaries. The authors clarified their finding that oocytes can be formed in a single day following peripheral blood cell transplantation: they proposed that germline stem cells from the bone marrow that are released into the peripheral blood stream are already somewhat differentiated, 
such that by the time they reach the ovary, their development into oocytes is accelerated. By contrast, oocyte regeneration took place over 2 months after bone marrow transplantation. Finally, in response to criticism regarding the applicability of their findings to human reproduction, Johnson et al. cited several cases of patients who received bone marrow or peripheral blood cell transplants following high-dose chemotherapy for hematopoietic reconstitution who also recovered ovarian function and fertility. They also cited several papers supporting the hypothesis that bone marrow germline stem cell senescence may be involved in ovarian aging, and may contribute to the loss of fertility in menopause.

\section{Eggs forever?}

Byskov AG, Faddy MJ, Lemmen JG, Andersen CY

Laboratory of Reproductive Biology, Juliane Marie Centre, Rigshospital, Copenhagen, Denmark agb.Irb@rh.dk

Differentiation 2005;73:438-446

Background: In this article, Anne Byskov examined the Tilly model of putative oocyte regeneration and could not confirm the presence of positive staining (SSEA) for primordial germ cells in the adult ovary, apparently refuting the contention that stem cells are recruited to the ovary.

A rigorous analysis of the neo-oogenesis/neo-folliculogenesis model is provided in this paper with a critique of methods and interpretation. This paper concludes that there is no evidence that new oocytes emerge in the adult animal and seemingly brings closure to the debate. The French physiologist Claude Bernard wrote: Men who have excessive faith in their theories or ideas are not only illprepared for making discoveries; they also make poor observations. Whether the world will remember the Byskov analysis or the Tilly paper remains to be seen.

\section{New paradigms: when testicular function starts to PACAP}

\section{Delayed testicular aging in pituitary adenylate cyclase-activating peptide (PACAP) null mice}

Lacombe A, Lelievre V, Roselli CE, Salameh W, Lue Y, Lawson G, Muller JM, Waschek JA, Vilain E

David Geffen School of Medicine, University of California, Gonda Center, Los Angeles, Calif., USA

evilain@ucla.edu

Proc Natl Acad Sci USA 2006;103:3793-3798

Background: The age-related decline in male sex hormone release is a direct consequence of testicular aging, but the mechanisms underlying this process are poorly understood. One potential mediator of this effect is PACAP, a polypeptide that regulates testicular steroidogenesis in vitro.

Methods: Testicular structure and function was compared between wild-type and $\mathrm{PACAP}^{-1-}$ male mice, at 4 and 15 months of age.

Results: Steroidogenesis (evaluated by levels of testosterone, steroidogenic acute regulatory protein, 3hydroxysteroid dehydrogenase, and $\mathrm{P}_{450} \mathrm{c} 17$ ) was impaired in $\mathrm{PACAP}^{-1-}$ mice at 4 months of age. However, the testicular structure of these animals was not affected. At 15 months of age, wild-type testis displayed typical signs of aging, whereas testicular structure was remarkably well conserved in PACAP $^{-l-}$ animals. The depletion of germ cells found in wild-type animals was associated with a higher content of peroxynitrites, a marker of reactive oxygen species, compared with $\mathrm{PACAP}^{-/-}$mice.

Conclusion: Testicular aging is delayed in $\mathrm{PACAP}^{-/-}$animals. One potential mechanism for this is a reduction in reactive oxygen species - a by-product of steroidogenesis - with consequently lower levels of apoptosis.

PACAP is well established as a regulator of signal transduction in the gonadotrope, but the mouse model of PACAP deletion reported here shows a marked testicular phenotype. The authors propose that 'testicular aging' is the main reason for decreased fertility and testosterone production during 
the andropause, and suggest that reactive oxygen species, a by-product of steroidogenesis, contribute to this process. Of note, other factors could influence testicular aging in this setting. It has been shown that PACAP ${ }^{-1-}$ mice present numerous phenotypes, including high mortality and reduction in glucose levels [6]. In addition, PACAP has some structural homology with vasoactive intestinal peptide (VIP) and in a more limited fashion with GHRH, although testicular GnRH receptor expression seems to play a less important role than originally proposed. Whether PACAP plays a major role in humans remains to be show. However, this study raises the possibility that accelerated or excessive testicular steroidogenesis may be associated with earlier gonadal senescence.

\section{New hope: ova to you}

\section{Ovarian transplantation between monozygotic twins discordant for premature ovarian failure}

Silber SJ, Lenahan KM, Levine DJ, Pineda JA, Gorman KS, Friez MJ, Crawford EC, Gosden RG

Infertility Center of St. Louis, St. Luke's Hospital, St. Louis, Mo., USA

drsherm@aol.com

N Engl J Med 2005;353:58-63

Background: Ovarian grafts have been shown to restore function and fertility in animal models; the possibility of ovarian tissue transplantation in humans has given hope to women who have diseases or received treatments that have rendered them sterile.

Methods: 24-year-old monozygotic twins presented with discordant ovarian function: one sister had normal fertility and the other sister had premature ovarian failure at age 14 . The fertile (donor) sister underwent laparoscopic removal of the left ovary, which was dissected ex vivo. Ovarian cortical tissue was trimmed to remove medullary tissue and small antral follicles. The recipient sister's ovary was resected to expose the surface of the medulla. Sections of the donor cortex were positioned over the exposed recipient medulla and sutured in place.

Results: The patients recovered rapidly; follicles developed in the recipient's ovary 71 days after transplantation and the recipient had her first menses 80 days after surgery. A second follicle developed 128 days after transplantation, with a second menses starting 142 days after surgery. The recipient's gonadotropin levels fell to within the normal range. Five weeks after the second menstrual period (day 176 after transplantation), ultrasound confirmed a normal uterine pregnancy that developed normally. The recipient gave birth to a healthy-appearing female infant at 38 weeks' gestation.

Conclusions: After receiving a transplant of ovarian cortical tissue from her fertile twin sister, the recipient resumed menses and conceived and delivered a healthy female infant. This case report demonstrated that ovarian function can be restored and that a successful pregnancy can be achieved after ovarian cortical transplantation.

Transplantation of reproductive tissue poses significant immunological challenges, which were avoided in this report of transplantation between monozygous twins. Although cortical transplantation between monozygotic twins with discordant fertility will likely be a rare event, this research represents a significant breakthrough in the effort to preserve or restore female fertility that has been lost to disease or the treatment of disease. Transplantation of the whole ovary is far more complicated and requires more recovery time than the simplified cortical transplantation procedure described in this study. Combined with advances in cryopreservation of ovarian tissue, this procedure may provide an unprecedented opportunity for autotransplantation of stored cortical tissue, thereby preserving fertility after sterilizing treatments, such as high-dose chemotherapy. 


\title{
Pregnancy after transplantation of cryopreserved ovarian tissue in a patient with ovarian failure after chemotherapy
}

\author{
Meirow D, Levron J, Eldar-Geva T, Hardan I, Fridman E, Zalel Y, Schiff E, Dor J
}

Chaim Sheba Medical Center, Tel Hashomer, Israel

meirow@post.tau.ac.il

N Engl J Med 2005;353:318-321

Background: Cryopreservation of tissue for later autotransplantation may allow the preservation of fertility in women who must undergo sterilizing chemotherapy.

Methods: Cortical ovarian tissue was collected from a 28-year-old woman after second-line conventional chemotherapy, prior to high-dose chemotherapy for non-Hodgkin's lymphoma. The patient subsequently experienced ovarian failure, with amenorrhea, high gonadotropin levels, and undetectable levels of anti-mullerian hormone and inhibin B that persisted for 2 years. Autotransplantation of the cryopreserved ovarian tissue was then performed, in which strips of thawed tissue were transplanted to the left ovary and small fragments were injected into the right ovary.

Results: Eight months after autotransplantation, the patient resumed menstruation with concomitant increases in anti-mullerian hormone and inhibin B. Gonadotropin levels fell to within the normal range by the second menses, which occurred 9 months after surgery. After the next modified natural cycle (using a $\mathrm{GnRH}$ antagonist), a single mature oocyte with a large cumulus was retrieved and fertilized; pregnancy was confirmed 12 days after embryo transfer. Fetal growth and development were normal and a healthy-appearing female infant was delivered by caesarean section at 38 weeks 5 days gestation. Conclusion: Cryopreservation of ovarian tissue and subsequent autotransplantation can restore fertility in women who have undergone sterilizing high-dose chemotherapy.

Previous reports have described fertilization of cryopreserved ovarian tissue in humans [7], and the birth of a normal baby following orthotopic transplantation of thawed ovarian tissue [8] to a woman post-chemotherapy. However, lingering doubts have remained in this second case as to whether the pregnancy was the result of spontaneous recovery of remaining ovarian tissue rather than from the transplanted cryopreserved material. This new report by Meirow et al. provides convincing evidence that ovarian cryopreservation can work. Despite this progress, cryopreservation of the whole ovary or ova has not been as successful as once hoped, but new techniques and media for storing ovarian tissue or follicles currently being developed by several groups may improve the outcome. These efforts seek to provide women, as well as young girls, the option to preserve fertility and the possibility of having children, even as they face potentially sterilizing diseases and therapies. Of course, these procedures come with a new set of ethical implications, including whether removal and storage of ovarian tissue should be considered an elective procedure or a medical necessity limited to a certain subset of patients. The application of this procedure may also raise concern if it requires a delay in chemotherapeutic treatment. Issues of informed consent, particularly with regard to young girls, may present a major obstacle to routine ovarian tissue banking. Nevertheless, these studies provide real hope to many women who are essentially facing the end of their reproductive lives even as their lives are prolonged through highly effective chemotherapy and radiation treatments. In the coming years, improvements in the techniques for the collection, storage, and autotransplantation of ovarian tissue are expected to continue. 


\title{
Success of testicular sperm injection and intracytoplasmic sperm injection in men with Klinefelter syndrome
}

\author{
Schiff JD, Palermo GD, Veeck LL, Goldstein M, Rosenwaks Z, Schlegel PN
}

The James Buchanan Brady Foundation, Department of Urology, and The Center for Reproductive Medicine and Infertility, The New York-Weill Cornell Medical Center, and the Population Council, New York, N.Y., USA pnschleg@med.cornell.edu

J Clin Endocrinol Metab 2005;90:6263-6267

Background: Testicular sperm extraction (TESE) and intracytoplasmic sperm injection (ICSI) have been successfully used for fertility treatment in men with Klinefelter syndrome (KS) but the exact benefits of this approach have not been examined in a large series.

Methods: A total of 42 men with KS who underwent 54 TESE procedures were identified. Before TESE, patients with serum testosterone levels $<15.6 \mathrm{nmol} / 1$ were treated with an aromatase inhibitor. Sperm retrieval rates and results of ICSI were analyzed.

Results: Spermatozoa were found during 39 microdissection TESE procedures, on the day before, or day of oocyte retrieval during a programmed in vitro fertilization cycle. The sperm retrieval rate was $72 \%$ (39 of 54) per TESE attempt, and 29 of the 42 different men (69\%) had adequate sperm found for ICSI. In vitro fertilization yielded embryos for transfer in $85 \%$ (33/39) cycles with sperm retrieved. Eighteen clinical pregnancies occurred, resulting in 21 live births [18 of $39(46 \%)$ ]. All children had a normal karyotype. Conclusion: TESE/ICSI is a successful intervention for the majority of patients with azoospermia and KS. Sperm retrieval and ICSI success in men with KS are comparable with other men with non-obstructive azoospermia treated in a specialist center.

\begin{abstract}
Although several case reports and small series have described the successful use of ISCI in men with nonmosaic KS, this is the first major series of combining TESE and ISCI in an attempt to obtain fertility in KS men from one specialized center. Considering the presence of a Sertoli cell-only pattern on diagnostic biopsy, the results are surprisingly good, with almost $70 \%$ of KS men having adequate sperm retrieval for ICSI, and successful birth of children with normal karyotypes. It is clear that excellent results can be obtained with such an invasive and intensive approach, involving a dedicated, focused and experienced multidisciplinary team. Whether similar results will be achievable in other units remains to be seen. Also, the authors suggest that the outcome appears to be worse in men who had puberty induced with highdose testosterone. Whether this is causative or associative is not clear (i.e., those with worse testicular function are more likely to require complete puberty induction). More data are needed in this regard. Furthermore, outcome may be worse with age, as shown by Okada et al. [9] in a study where success rate of TESE in non-mosaic KS fell from $81 \%$ in the 25 - to 29 -year-old age group to $22 \%$ between 40 and 44 years. Taken together, these studies suggest that fertility prospects in KS appear much brighter, especially when attempted in young adulthood. The pediatric endocrinologist will have an important role to play in counseling patients and optimizing testosterone replacement in the peripubertal period.
\end{abstract}

\section{Cryopreservation of intact testicular tissue from boys with cryptorchidism}

Kvist K, Thorup J, Byskov AG, Hoyer PE, Mollgard K, Yding Andersen C

Laboratory of Reproductive Biology, Department of Paediatric Surgery, University Hospital of Copenhagen,

Rigshospitalet, Copenhagen, Denmark

yding@rh.dk

Hum Reprod 2006:21:484-491

Background: Boys with cryptorchidism are often infertile in adulthood despite having orchiopexy performed at a very young age. A biopsy of the testis is often taken during this procedure to evaluate testicular morphology and to examine for the presence of neoplasia. However, the capacity for cryopreserved testicular biopsies to be used for possible fertility preservation is not known.

Methods: Testicular biopsies were obtained from 8 boys (11 samples) at the time of surgery. Each sample was divided into six parts. Two fresh samples were cultured for 2 weeks. Four samples were cryopreserved (for $1 \mathrm{~h}$ ) then thawed and cultured for 2 weeks. 
Results: The morphology of fresh and frozen-thawed samples was similar, with well-preserved seminiferous tubules and interstitial cells. A similar picture appeared after 2 weeks of culture, but a few of the cultured biopsies contained small necrotic areas. The presence of spermatogonia was verified by immunostaining. Testosterone and inhibin B production in the frozen-thawed pieces was on average similar to that of the fresh samples.

Conclusion: Intact testicular tissue from young boys with non-descended testes tolerates cryopreservation with surviving spermatogonia and without significant loss of the ability to produce testis-specific hormones in vitro. Part of a testicular biopsy routinely taken at the time of orchidopexy might have potential for fertility preservation in adult life.

\begin{abstract}
Preservation of reproductive function is 'flavor of the month' and, following media coverage and expectations of what might be possible in the future, there is increasing pressure from parents to consider testicular cryopreservation when biopsies are taken in childhood, or even when gonads are removed in 46,XY patients with partial gonadal dysgenesis or steroid biosynthetic defects. Will immature testicular tissue be of any use for fertility preservation in years to come? The answer is not yet known, but in this short-term cryopreservation study Kvist et al. show that testicular steroidogenesis and germ cell integrity is preserved following a freeze-thaw cycle followed by culture. This is an important potential approach, but would need serious consideration of the long-term ethical and biological issues before being adopted widely. Unfortunately, it could be some time before it is known whether modern approaches to cryopreservation will yield useful tissue many years from now.
\end{abstract}

New concerns: congenital disorders only become apparent in childhood

\title{
Hypospadias in a cohort of 1,072 Danish newborn boys: prevalence and relationship to placental weight, anthropometrical measurements at birth, and reproductive hormone levels at three months of age
}

Boisen KA, Chellakooty M, Schmidt IM, Kai CM, Damgaard IN, Suomi AM, Toppari J, Skakkebaek NE, Main KM University Department of Growth and Reproduction, Rigshospitalet, Copenhagen, Denmark katharina.main@rh.hosp.dk

J Clin Endocrinol Metab 2005;90:4041-4046

Background: Hypospadias is one of the most frequent male congenital malformations and may be part of the testicular dysgenesis syndrome. The true prevalence of hypospadias and its relation to anthropometrical measurements at birth and reproductive hormone levels at 3 months of age is still debated. Methods: A prospective cohort study of 1,072 Danish boys recruited antenatally with 3-year follow-up (1997-2004) was performed, with $74.4 \%$ boys completing the study. The position of the urethral meatus, anthropometrical measurements, placental weight, and reproductive hormone levels were measured. Results: The Danish prevalence of hypospadias at birth was $1.03 \%$. At 3 years, the true prevalence was found to be $4.64 \%$ because additional mild cases were detected when physiological phimosis dissolved. Weight for gestational age and placental weight were significantly lower, and FSH was significantly higher in boys with hypospadias, compared with healthy boys.

Conclusions: A surprisingly high total rate of hypospadias of $4.6 \%$ was found in this large prospective cohort study. Seventy-two percent of the cases were apparent only after the prepuce could be retracted. An association between fetal growth impairment and hypospadias was confirmed. However, it is not yet known whether this represents a causal relationship or a shared pathogenic factor.

This study reports an alarmingly high prevalence of hypospadias in the Danish population and - most importantly - that this feature may only be apparent once the prepuce can be retracted. Thus, previous reports of the prevalence of hypospadias in the newborn may be an underestimate of the true prevalence of this condition. This study also confirms the association between fetal growth impairment and male genital abnormalities. This association is poorly understood at present, and it remains unclear whether it is causal or associative. Indeed, most children with $46, \mathrm{XY}$ disorders of sex development (e.g. underandrogenization) who are born with intrauterine growth retardation do not have a 
cause found for their condition and analysis of the most frequent testis determining genes usually proves fruitless.

\section{Concepts revised: upregulating the $X$}

\section{Dosage compensation of the active $X$ chromosome in mammals}

Nguyen DK, Disteche CM

Department of Pathology, University of Washington, Seattle, Wash., USA

cdistech@u.washington.edu

Nat Genet 2006;38:47-53

Background: In mammals, the presence of a single X chromosome in the male and two X chromosomes in the female means that dosage compensation mechanisms are necessary to restore balanced expression between the X chromosome(s) and autosomes. In Drosophila, upregulation of the male X leads to dosage compensation. It has been hypothesized that mammals likewise upregulate their active X chromosome and, together with $\mathrm{X}$ inactivation, this mechanism would maintain balanced expression between the $\mathrm{X}$ chromosome and autosomes and between the sexes.

Methods: Analysis of X-chromosomal expression in different tissues from various mammalian species.

Results: Doubling of the global expression level of the $\mathrm{X}$ chromosome leads to dosage compensation in somatic tissues from several mammalian species, especially in the brain. Of note, the X chromosome is expressed but not upregulated in spermatids and secondary oocytes, preserving balanced expression of the genome in these haploid cells.

Conclusion: Upon fertilization, upregulation of the active $\mathrm{X}$ occurs to achieve dosage compensation in early embryos.

Our traditional concept is that one of the $\mathrm{X}$ chromosomes undergoes inactivation in females, so that a balance between $X$ chromosome genes expression is maintained between the sexes. This iconoclastic study by Nguyen and Disteche reported in Nature Genetics proposes that upregulation of an active $X$-chromosome occurs in somatic tissues of both sexes to maintain the balance not just between the sex chromosomes in males and females, but also between sex chromosomes and autosomes. This process may be particularly important in structures such as the brain, whereas upregulation of the active $X$ does not occur in haploid tissues, such as gametes. It remains to be seen whether variation in X-activation, as well as X-inactivation, can account for phenotypic variability in Turner syndrome, variable penetrance in carriers of X-linked conditions, or whether our concepts of gene dosage mechanisms involving $X$-chromosome genes in endocrine development (e.g. DAX1, gonad; SOX3, pituitary; SHOX, growth plate) might need reconsideration in light of this study.

\section{Important for clinical practice: The postnatal testosterone surge in boys has clinical and biochemical correlates}

\section{Postnatal penile length and growth rate correlate to serum testosterone levels: a longitudinal study of 1,962 normal boys}

Boas M, Boisen KA, Virtanen HE, Kaleva M, Suomi AM, Schmidt IM, Damgaard IN, Kai CM, Chellakooty M, Skakkebaek NE, Toppari J, Main KM

University Department of Growth and Reproduction, Rigshospitalet, Copenhagen, Denmark

katharina.main@rh.hosp.dk

Eur J Endocrinol 2006;154:125-129

Background: The physiological significance of the brief hypothalamic-pituitary-gonadal axis activation observed shortly after birth in boys is unknown, as is the relation between endogenous testosterone levels at this time and penile size and growth. 
Methods: Infant boys (728 Danish, 1,234 Finnish) were examined clinically at 0, 3, and 18 months (and at 36 months too in Denmark) with blood samples taken at 3 months in 630 boys. Penile length and growth were recorded and reproductive hormones (testosterone, sex hormone binding globulin, estradiol) were analyzed.

Results: Penile length increased from birth $(3.49 \pm 0.4 \mathrm{~cm})$ to 3 years of age $(4.53 \pm 0.51 \mathrm{~cm})$ with the highest growth velocity from birth to 3 months $(1.0 \mathrm{~mm} / \mathrm{month})$. Penile length and growth were significantly, positively correlated with serum testosterone and with free testosterone index.

Conclusion: Endogenous testosterone is significantly associated with penile size and growth rate in infant boys. Thus, the postnatal surge in reproductive hormones appears to be important for genital growth. These data also serve as an updated reference for normal penile length in Caucasian boys up to 3 years of age.

The presence of the postnatal testosterone surge in male infants has been known for 30 years [10]. However, it is unclear whether this rise in testosterone peaking at around 3 months of age has any true role in physiological function or even contributing to subsequent male gender identity or sex role behavior. To address this in an associative study, Boas et al. have shown a correlation between testosterone at 3 months of age and postnatal penile length and penile growth in a study of 1,962 male infants. Thus, androgen-dependent mechanisms seem to be important in penile growth within the population. Whilst other factors may influence androgen responsiveness (e.g. trinucleotide repeats in the androgen rector, patterning genes involved in penile development), this study does highlight that the postnatal surge is associated with androgen responsiveness, which may reflect the spectrum of androgenic activity in utero. Some practical aspects arise from this study: (i) this period of infancy may be used as a limited time window to establish the diagnosis of hypogonadotropic hypogonadism or testicular failure and (ii) here we have new normal longitudinal reference curves for penile growth, in Denmark at least.

\section{Prevalence and incidence of precocious pubertal development in Denmark: an epidemiologic study based on national registries}

Teilmann G, Pedersen CB, Jensen TK, Skakkebaek NE, Juul A

Department of Growth and Reproduction, Rigshospitalet, University of Copenhagen, Copenhagen, Denmark gteilmann@rh.dk

Pediatrics 2005;116:1323-1328

Background: No population-based epidemiologic studies on the incidence and prevalence of precocious pubertal development have been published. Danish national registries provide sufficient data for estimating the prevalence and incidence of this condition.

Methods: The age- and gender-specific incidence rates as well as prevalence rates of precocious pubertal development in Denmark were estimated using data from the Danish National Patient Registry and Statistics Denmark over a 9-year period (1993-2001).

Results: Overall, 670 children were registered with a diagnosis of precocious puberty (PP) from 1993 to 2001, corresponding to 50-70 new cases of PP per year in Denmark. The incidence of PP was constant during the study period from 1993 to 2001 . The incidence of PP subdivided by gender and age at diagnosis was approximately 0.5 per 10,000 in girls under 2 years, below 0.05 per 10,000 in girls between the ages of 2-4 years, and rising gradually to 8 per 10,000 in girls aged 5-9 years. For boys, the incidence was very low under 8 years of age $(<1$ per 10,000) and increased only slightly to $1-2$ per 10,000 in boys aged 8-10 years. The prevalence of PP was approximately $20-23$ per 10,000 girls, whereas the prevalence was 5 -fold lower for boys $(<5$ per 10,000).

Conclusions: This first epidemiologic study based on national registries has estimated that $0.2 \%$ of all Danish girls and $<0.05 \%$ of Danish boys had some form of precocious pubertal development.

Although it is often publicized that puberty is getting earlier, especially in girls, there is still considerable debate about whether this is just an advance on the onset of breast development or whether the entire pubertal process occurs at a younger age. Furthermore, significant differences occur in different populations and racial groups. Thus, it is very important to obtain accurate population-based data on pubertal development, so that future studies can have a data with which to compare their 
findings. This report by Teilmann et al. shows no changes in the incidence of precocious puberty over the 9-year study period, and provides exactly the kind of accurate population-based study against which longer term changes in puberty onset can be assessed.

\section{Truth-telling and Turner syndrome: the importance of diagnostic disclosure}

Sutton EJ, Young J, McInerney-Leo A, Bondy CA, Gollust SE, Biesecker BB

National Human Genome Research Institute and the National Institute of Child Health

and Human Development, National Institutes of Health, Bethesda, Md., USA

barbarab@mail.nih.gov

J Pediatr 2006;148:102-107

Background: Non-disclosure or 'secret-keeping' was historically common with conditions such as Turner syndrome (TS). Perceived effects of withholding information have not been analyzed extensively.

Methods: Girls and women $(\mathrm{n}=97)$ and 21 parents participated in the initial interview study. Transcripts were coded and analyzed for constructs related to secret-keeping.

Results: Thirty percent of participants spontaneously mentioned that their health care providers (HCP) or parents had withheld all or part of their TS diagnosis. Of those, 15 individuals were not informed of the infertility component of their diagnosis. Individuals reporting secret-keeping were more likely to have had a negative perception of the HCP's role in the disclosure process compared with those participants who did not report that a secret had been kept $(\mathrm{p}<0.025)$.

Conclusion: The prevalence of secret-keeping within this sample population suggests it is likely a significant concern in the greater TS population. How HCPs disclose a TS diagnosis may affect whether secrets are kept. Conversely, secret-keeping may result in a negative disclosure experience. These observations suggest the need for interventions aimed at helping HCPs disclose health-related information to parents and their children in a timely, caring, and sensitive manner.

\section{$X Y$ females: revisiting the diagnosis}

Minto CL, Crouch NS, Conway GS, Creighton SM

The Middlesex Clinic, Elizabeth Garrett Anderson Hospital, University College London Hospitals, London, UK BJOG 2005;112:1407-1410

Background: The assigned diagnosis in XY female intersex conditions may be less accurate than originally thought in many individuals who are now in adulthood.

Methods: A cross-sectional review was performed of the hospital case notes of 46 adult 'intersex' women with a complete or mosaic XY karyotype. Details of presentation, all investigations, subsequent clinical course and all treatments (medical and surgical) were analyzed. An 'up-to-date' diagnosis was generated and compared to the recorded or working diagnosis.

Results: An accurate diagnosis was found in $47.8 \%$ of patients. In $32.6 \%$ the diagnoses were inaccurate and currently under review, $13 \%$ had a wrong diagnosis and $6.5 \%$ remain with an unknown etiology. Conclusions: This study shows $52.1 \%$ of patients have unknown, inaccurate or wrong diagnoses. Assigning the wrong diagnosis may be harmful, for example, if it leads to irreversible virilizing changes or development of a gonadal malignancy. Diagnostic accuracy aids appropriate management and genetic counseling for both the patient and their immediate family.

These two reports clearly show the importance of pediatric endocrinologists getting it right with the diagnosis, disclosure and counseling of conditions such as TS and disorders of sex development. 


\section{Targets of microRNA regulation in the Drosophila oocyte proteome}

Nakahara K, Kim K, Sciulli C, Dowd SR, Minden JS, Carthew RW

Department of Biochemistry, Molecular Biology, and Cell Biology, Northwestern University, Evanston, III., USA

r-carthew@northwestern.edu

Proc Natl Acad Sci USA 2005;102:12023-12028

Background: MicroRNAs (miRNAs) are short RNA sequences ( $\sim 22 \mathrm{nt}$ long) that are involved in gene silencing by binding to $3^{\prime}$-untranslated regions of specific target mRNA and interfering with translation. Although most miRNA genes are conserved between related species and $\sim 30 \%$ have orthologs in vertebrates and invertebrates, the gene targets of miRNAs remain largely unknown.

Methods: A proteomic screen was conducted to identify miRNA target genes within late-stage Drosophila oocytes from dcr-1 null mutants. Dcr-1 encodes the Dicer enzyme that is essential for miRNA biogenesis in Drosophila; miRNA targets were identified as those that were derepressed in the proteome of $d c r-1$ mutant flies compared with wild-type flies. Target proteins were then identified by mass spectrometry.

Results: 41 of 1,003 proteins $(4 \%)$ were enriched in the $d c r-1$ mutants as compared with wild-type flies. The fold increase ranged from 1.6- to 69-fold. Most of the proteins were found to function in protein metabolism, including protein synthesis, folding, intracellular transport, and turnover. A few of the $d c r-1$-enriched proteins were involved in carbohydrate and lipid metabolism, and a few had unknown functions. Transcripts for 18 of the 41 proteins were found to have similar abundance in the $d c r-1$ and wild-type flies, suggesting that miRNA may be involved in the translational repression of gene expression.

Conclusions: The repression of translation of mRNA that encodes proteins necessary for protein biogenesis and turnover may be necessary in late-stage Drosophila oocytes in order to allow accumulation and steady-state maintenance of proteins necessary for embryogenesis.

A significant, lingering mystery in the field of developmental biology is the mechanism by which oocytes are able to direct early developmental processes. With virtually nothing but DNA contributed by the male at fertilization, the egg must have ready the necessary proteins to drive resumption of meiosis, initiate ultrastructural changes, permit pronuclear fusion, and support subsequent mitosis and cell division. These proteins must be maintained for an indefinite and variable period of time until fertilization occurs. The concept that RNA silencing may be involved in maintaining oocytes and their protein complement in this static state is intriguing. It is also interesting that only 41 of 1,003 proteins (or $4 \%$ of genes) might be directly inhibited. Beyond a glimpse into the potential mechanism underlying oocyte activation, these data also bring us a step closer to identifying the proteins involved in this process.

\section{New genes: different expressions between the sexes}

\section{Expression profiling of purified mouse gonadal somatic cells during the critical time window of sex determination reveals novel candidate genes for human sexual dysgenesis syndromes}

Beverdam A, Koopman P

Division of Genetics and Developmental Biology, Institute for Molecular Bioscience, The University of Queensland, Brisbane, Australia

p.koopman@imb.uq.edu.au

Hum Mol Genet 2006;15:417-431

Background: Despite the identification of SRY as the testis-determining gene in mammals, the genetic interactions controlling the earliest steps of male sex determination remain poorly understood. In particular, 
the molecular lesions underlying a high proportion of human XY gonadal dysgenesis, XX maleness and XX true hermaphroditism remain undiscovered.

Methods: Using a novel reporter mouse line that expresses enhanced green fluorescent protein under the control of a Sf1 promoter fragment, Sertoli and granulosa cell precursors were marked during the critical period of sex determination. These cells were purified from gonads of male and female transgenic embryos at $10.5 \mathrm{dpc}$ (shortly after Sry transcription is activated) and $11.5 \mathrm{dpc}$ (when Sox 9 transcription begins), and their transcriptomes analyzed using Affymetrix genome arrays.

Results: In $11.5 \mathrm{dpc}$ male somatic gonad cells, 266 upregulated genes (including Dhh, Fgf9 and Ptgds) and 50 downregulated genes were identified. In $11.5 \mathrm{dpc}$ female somatic gonad cells, 242 genes (including follistatin) were upregulated. The majority of these genes are novel genes that lack identifiable homology, and several human orthologs were found to map to chromosomal loci implicated in disorders of sexual development.

Conclusions: These genes represent an important resource with which to piece together the earliest steps of sex determination and gonad development, and provide new candidates for mutation searching in human sexual dysgenesis syndromes.

\section{Gene expression during sex determination reveals a robust female genetic program at the onset of ovarian development}

Nef S, Schaad O, Stallings NR, Cederroth CR, Pitetti JL, Schaer G, Malki S, Dubois-Dauphin M, Boizet-Bonhoure B, Descombes P, Parker KL, Vassalli JD

Department of Genetic Medicine and Development, University of Geneva Medical School, Geneva, Switzerland Serge.Nef@medecine.unige.ch

Dev Biol 2005;287:361-377

Background: The primary event in mammalian sexual development is the differentiation of the bipotential gonads into either testes or ovaries, but the initial molecular changes accompanying gonadal differentiation in mice are poorly understood.

Methods: A large-scale transcriptional analysis of XX and XY Sf1-positive gonadal cells during sex determination was performed.

Results: In both male and female genital ridges, a robust genetic program is initiated pre-dating the first morphological changes of the differentiating gonads. Between E10.5 and E13.5, 2,306 genes were expressed in a sex-specific manner in the somatic compartment of the gonads; 1,223 were overexpressed in XX embryos and 1,083 in XY embryos. Although sexually dimorphic genes were scattered throughout the mouse genome, chromosomal regions containing clusters of genes displaying similar expression profiles were identified.

Conclusions: These studies define the major characteristics of testicular and ovarian transcriptional programs and reveal the importance of signaling processes in differentiation of the bipotential gonads into testes and ovaries.

Although several single genes have been identified that play a key role in early testis development (e.g. SRY, SOX9), many of the genes that are differentially expressed at critical periods of gonad (testis vs. ovary) development remain elusive. Microarray technology holds great potential in this regard, but the problem with this approach in mice has been the small amounts of RNA available from the key structures during early development, and the ability to focus on just those cells becoming committed to gonadal development from the mass of supportive parenchymal cells, which would 'dilute' any key signal. By combining RNA amplification methods with cell sorting based on expression of steroidogenic factor-1, these two reports have identified clusters of genes expressed at early stages of gonad determination. Many of these factors are involved in signaling pathways, and it seems that the ovary has a much more robust pattern of gene expression than previously thought. Ovarian development is no longer a 'default' event. It is likely that many of these genes will be involved in human gonad development and can provide candidates for gonadal dysgenesis syndromes in humans. However, it is also clear than many of the mechanisms between humans and mice are different, and elucidating these basic differences will be fascinating in due course. 


\section{The structure of the follistatin:activin complex reveals antagonism of both type I and type II receptor binding}

Thompson TB, Lerch TF, Cook RW, Woodruff TK, Jardetzky TS

Department of Biochemistry, Northwestern University, Evanston, III., USA

tedj@northwestern.edu

Dev Cell 2005;9:535-543

Background: Activin is inhibited by the binding of extracellular follistatin. Previous studies have suggested that follistatin binding to activin blocks the binding site for the type II receptor.

Methods: The structure of activin in complex with follistatin has been solved to $2.8 \AA$ and was compared to a previously solved structure of activin in complex with the activin type IIB receptor to elucidate the mechanism of activin inhibition.

Results: Two follistatin molecules bind to the activin dimer and block the sites involved in binding by both the activin type II and type I receptors.

Conclusions: The follistatin-activin crystal structure provides insight into the differential interaction of follistatin with various members of the TGF $\beta$ superfamily, and the potential role of follistatin in various TGF $\beta$ superfamily-mediated biological processes.

Follistatin was first identified as a potent inhibitor of activin, myostatin and several bone morphogenic proteins (BMPs). The many ligands, receptors, and inhibitors of the TGF $\beta$ superfamily regulate diverse and distinct physiologic processes, yet there is some level of promiscuity between the various signaling components. For example, the BMP ligands can bind to activin receptors, and membrane-bound $\beta$-glycan has been shown to inhibit activin and BMP signaling while enhancing TGF $\beta$ signaling. Likewise, follistatin inhibits the activity of several TGF $\beta$ superfamily members, including BMP, though with much lower potency than it does activin. Follistatin has been shown to form a co-complex with both BMP and its type I signaling receptor, BMP-RI. In contrast, the BMP inhibitor noggin binds to BMP and blocks access to the type I and type II receptor binding sites. Similarly, the authors found that follistatin inhibits activin by blocking access to both the type I and type II receptor binding sites. These data provide structural evidence of different mechanisms of inhibition by follistatin and other inhibitor proteins against individual TGF $\beta$ superfamily ligands. Furthermore, these data reveal that follistatin inhibition of activin involves the blockade of the activin type I receptor binding site, suggesting that the type I receptor may play a more significant role in initial activin binding to its receptor complex than once thought. Whilst not a 'new hormone' as such, follistatin is emerging as an important regulator of reproductive development and function (e.g. early ovarian development), and the follistatin-activin system may prove to have important clinical consequences for the pediatric endocrinologist in the future.

\section{Review: hair today, gone tomorrow}

\section{Clinical practice. Hirsutism}

Rosenfield RL

Department of Pediatrics and Medicine, University of Chicago, Pritzker School of Medicine, Chicago, III., USA robros@peds.bsd.uchicago.edu

N Engl J Med 2005;353:2578-2588

A very useful review for a difficult problem being seen increasingly by the pediatric and adolescent endocrinologist. This review highlights: (1) the subjectivity of assessment, (2) the challenges of interpreting cyclical and episodic variation in ovarian and adrenal function, and (3) the problems of interpreting data in the absence of reliable total and free testosterone assays with well-validated normal ranges for women without hirsuitism. These challenges can be particularly pronounced when dealing with the adolescent cohort. 


\title{
Transient testicular warming enhances the suppressive effect of testosterone on spermatogenesis in adult cynomolgus monkeys (Macaca fascicularis)
}

\author{
Lue Y, Wang C, Liu YX, Hikim AP, Zhang XS, Ng CM, Hu ZY, Li YC, Leung A, Swerdloff RS
}

Division of Endocrinology and Metabolism, Harbor-University of California, Los Angeles Medical Center, Torrance,

Calif., USA

swerdloff@labiomed.org

J Clin Endocrinol Metab 2006;91:539-545

Background: Testosterone $(\mathrm{T})$ and heat $(\mathrm{H})$ treatment have both been suggested as possible methods for suppressing spermatogenesis but it is not known if their effects are additive or synergistic.

Methods: A randomized, placebo-controlled study of adult cynomolgus monkeys treated for 12 weeks with: (1) two empty implants (C); (2) two T implants (T); (3) daily testicular heat exposure (43 C for $30 \mathrm{~min})$ for 2 consecutive days $(\mathrm{H})$, or (4) two $\mathrm{T}$ implants plus testicular heat exposure $(\mathrm{T}+\mathrm{H})$. Treatment was followed by an 8 -week recovery period.

Results: Sperm counts were transiently suppressed after heat to $16.4 \%$ of baseline at 4 weeks and then returned to pretreatment levels. Sperm counts were suppressed slowly after T treatment to a nadir of $6.4 \%$ of pretreatment levels at 12 weeks. $\mathrm{T}+\mathrm{H}$ rapidly suppressed sperm output as early as 4 weeks to $3.9 \%$ of pretreatment levels that was maintained throughout treatment. The decreased sperm counts were due to increased germ cell apoptosis in all treatment groups. Sperm counts recovered to the pretreatment levels in all groups by 8 weeks after treatment.

Conclusion: Transient testicular warming enhances and hastens the effect of $\mathrm{T}$ implant on the suppression of spermatogenesis in monkeys.

Despite significant advances in reproductive endocrinology over recent years, an effective malebased contraceptive remains elusive, and the majority of the world's population has limited access to simple, efficacious and safe birth control methods. In general, testosterone-based contraceptive approaches in males are proving disappointing but - as shown in monkeys by Lue et al. - combining this with intermittent testicular warming may have a greater effect on the suppression of spermatogenesis. This once again highlights the teleological role of the scrotum in higher mammals, and the potential pros and cons of hot baths!

Acknowledgment

J.C.A. holds a Wellcome Trust Clinician Scientist Fellowship (068061).

References

1. Stingl J, Eirew P, Ricketson I, Shackleton M, Vaillant F, Choi D, et al: Purification and unique properties of mammary epithelial stem cells. Nature 2006;439:993-997.

2. Johnson J, Canning J, Kaneko T, Pru JK, Tilly JL: Germline stem cells and follicular renewal in the postnatal mammalian ovary. Nature 2004;428:145-150.

3. Vogel G: Reproductive biology. Controversial study finds an unexpected source of oocytes. Science 2005;309:678-679.

4. Ainsworth C: Bone cells linked to creation of fresh eggs in mammals. Nature 2005;436:609.

5. Powell K: Skeptics demand duplication of controversial fertility claim. Nat Med 2005;11:911.

6. Cummings KJ, Pendlebury JD, Sherwood NM, Wilson RJ: Sudden neonatal death in PACAP-deficient mice is associated with reduced respiratory chemoresponse and susceptibility to apnoea. J Physiol 2004;555:15-26.

7. Oktay K, Buyuk E, Veeck L, Zaninovic N, Xu K, Takeuchi T, et al: Embryo development after heterotopic transplantation of cryopreserved ovarian tissue. Lancet 2004;363:837-840.

8. Donnez J, Dolmans MM, Demylle D, Jadoul P, Pirard C, Squifflet J, et al: Livebirth after orthotopic transplantation of cryopreserved ovarian tissue. Lancet 2004;364:1405-1410.

9. Okada H, Goda K, Yamamoto Y, Sofikitis N, Miyagawa I, Mio Y, et al: Age as a limiting factor for successful sperm retrieval in patients with nonmosaic Klinefelter's syndrome. Fertil Steril 2005;84:1662-1664.

10. Forest MG, Sizonenko PC, Cathiard AM, Bertrand J. Hypophyso-gonadal function in humans during the first year of life. 1. Evidence for testicular activity in early infancy. J Clin Invest 1974;53:819-828. 
